\title{
Digital Baseband Compensation of Joint TX/RX Frequency-Dependent I/Q Imbalance in Mobile MIMO-OFDM Transceivers
}

\author{
Balachander Narasimhan, Sudharsan Narayanan, Naofal Al-Dhahir, Hlaing Minn \\ Dept. of Electrical Engineering, University of Texas at Dallas
}

\begin{abstract}
Direct-conversion Orthogonal Frequency Division Multiplexing (OFDM) systems suffer from transmit and receive analog processing impairments such as Inphase/Quadrature (I/Q) imbalance causing Inter-Carrier Interference (ICI) between the sub-carriers. Another source of performance-limiting ICI, but with a different nature, in OFDM systems is Doppler spread due to mobility. Unlike previous work which considered these two problems separately, we develop a generalized analytical framework to characterize, estimate and jointly mitigate ICI due to both $I / Q$ imbalance and high mobility. Based on our general model, we exploit the special ICI structure to design efficient channel and I/Q imbalance parameter estimation and digital baseband compensation schemes for joint transmit/receive frequency-independent and frequencydependent $I / Q$ imbalance under high-mobility conditions. Moreover, we extend the model, compensation and channel estimation methods to the Multiple Input Multiple Output (MIMO) case, Spatial Multiplexing (SM) in particular.
\end{abstract}

Index Terms-OFDM, MIMO, I/Q imbalance, MMSE, Equalization, Mobility, ICI

\section{INTRODUCTION}

Orthogonal Frequency Division Multiplexing (OFDM) has been widely adopted as the transmission scheme of choice for almost all broadband wireless standards (including WLAN, WiMAX, LTE, DVB, etc.) due to its multipath resilience and practical implementation complexity. Traditionally, OFDM-based wireless transceivers used the super heterodyne architecture where the RF signal is converted down to baseband through 1 or 2 intermediate frequency (IF) stages. At each analog IF stage, filtering and amplification are applied to maintain acceptable signal selectivity and sensitivity. The main drawback of this architecture is the large number of ana$\log$ components (filters and amplifiers) needed to achieve acceptable signal quality which add to the overall power consumption and cost. To overcome these drawbacks, the direct-conversion architecture (where the RF signal is converted directly to baseband) has gained increased popularity recently since it enables low-cost low-power

Email: \{bxn062000,sxn062100,aldhahir,Hlaing.Minn\}@utdallas.edu Inc

This work was supported in part by a gift from Texas Instruments integration in CMOS technology leading to a smaller form factor. However, direct-conversion OFDM-based broadband wireless transceivers suffer from several major RF/analog impairments which limit their performance including $\mathrm{I} / \mathrm{Q}$ imbalance.

I/Q Imbalance refers to mismatches in amplitude and phase between the in-phase (I) and quadrature (Q) branches. Ideally, the I and Q branches of the mixers should have equal amplitude and $90^{\circ}$ phase shift but this is rarely the case in practice, resulting in frequencyindependent I/Q imbalance. Mismatches in other analog components such as transmit/receive analog filters and D/A or A/D converters could cause frequency-dependent I/Q imbalance.

Under high-mobility conditions, the sub-carriers in an OFDM symbol lose their orthogonality (due to Doppler effect), resulting in performance-limiting ICI which becomes more severe at higher speeds, higher carrier frequencies and larger OFDM block durations (necessary to combat severe channel frequency selectivity). Effective ICI mitigation requires accurate channel estimation which becomes very challenging at high speeds (where the channel is fast time-varying) especially for MIMO systems due to the increased number of channel parameters to be estimated while the total transmit power (from all antennas) stays the same as in single input single output (SISO) systems.

Recent advances in digital processing capabilities and VLSI technology scaling, fuelled by Moore's law, have widened the gap between digital and analog circuits in terms of their performance/complexity/cost tradeoffs. This trend is projected to become even more significant in the future. Furthermore, RF impairments in the analog components are mainly due to fabrication process variations which are difficult to predict or control, increase with the down-scaling of fabrication technologies, and can not be efficiently or completely cancelled in the analog domain due to power-area-cost tradeoffs.

All of the above-mentioned issues, coupled with the fact that embedded digital processors and custom ASICs in mobile devices are becoming more powerful, mo- 
tivate this research which aims at developing highperformance low-complexity digital baseband compensation techniques for I/Q imbalance in mobile OFDM systems.

Digital compensation of $\mathrm{I} / \mathrm{Q}$ imbalance in OFDM systems has been investigated in several recent papers [1]-[8]. Previous work in this area considers either I/Q imbalance or mobility. However, in broadband outdoor wireless systems (such as WiMAX,3GPP LTE or DVB$\mathrm{H})$, it is likely that both I/Q imbalance and mobility are present. This work is distinct from previous research in this area in that we develop a generalized mathematical model to quantify and compensate for the joint ICI effects of both impairments in the case where I/Q imbalance is frequency-dependent and occurs in both the transmit and receive ends. This generalizes our previous work in [9] which considered only frequency-independent I/Q imbalance at the receiver side. Furthermore, we exploit the channel and ICI structure to reduce the complexity of the compensation algorithms. We propose a joint channel and I/Q parameter estimation scheme that aids the effective compensation of the impairments. Moreover, we extend the proposed compensation and estimation methods to MIMO spatial multiplexing (SM) scheme.

This paper is organized as follows. In Section-II, we develop the generalized I/Q imbalance model. In Sections-III and -IV, we develop the low complexity compensation and channel estimation respectively. In Section-V, we extend the model and compensation algorithms to MIMO-OFDM systems. In Section-VI, we present the simulation results and the paper is concluded in Section-VII.

Notation: Functions $x(\cdot)$ are denoted by lower-case letters. All time-domain quantities $\{\bar{x}(t), \overline{\mathbf{x}}, \overline{\mathbf{X}}\}$ have a bar whereas frequency-domain quantities $\{x(f), \mathbf{x}, \mathbf{X}\}$ do not. Vectors $\{\overline{\mathbf{x}}, \mathbf{x}\}$ are represented by lower-case boldface. Matrices $\{\overline{\mathbf{X}}, \mathbf{X}\}$ are represented by upper-case boldface letters. $(\cdot)^{H}$ denotes the Hermitian, i.e. conjugate transpose of a matrix or a vector. The conjugate of a matrix, a vector, or a scalar is denoted by $(\cdot)^{*}$ and the transpose of a matrix or a vector is denoted by $(\cdot)^{T} . N$ is the size of the Discrete Fourier Transform (DFT). F is the unitary DFT matrix whose $(n, k)$ element is given by $\mathbf{F}_{n, k}=\frac{1}{\sqrt{N}} e^{-j \frac{2 \pi}{N} k n}$ with $0 \leq n, k \leq N-1$. Linear convolution is denoted by $\otimes$ while circular convolution modulo- $N$ is denoted by $\circledast N_{N}$. $\mathbf{I}_{k}$ is the identity matrix of size $k . \mathbf{0}_{m \times n}$ represents the all-zero matrix of size $m \times n$. The operator $\operatorname{Diag}(\cdot)$, when applied to a matrix results in a vector containning the diagonal elements of the matrix and when it acts on a vector results in a diagonal matrix whose diagonal elements are the elements of the vector. All signals are indexed modulo$N$, i.e., $x(k)=x((N-k))_{N} . \Re\{$.$\} and \Im\{$.$\} denote$ the real and imaginary parts of a complex number, respectively. $E\{\cdot\}$ is the expectation operator and $\delta(\cdot)$ is the dirac-delta function.

\section{I/Q IMBALANCE MODELING}

In this section, we derive a generalized mathematical model for joint $\mathrm{Tx} / \mathrm{Rx} \mathrm{I} / \mathrm{Q}$ imbalance and mobility in OFDM transceivers. Referring to Fig. 1, and following the convention that the subscript ' $t$ ' represents transmitside parameters and subscript ' $r$ ' represents receive-side parameters, we define the following

- $\alpha_{t}, \alpha_{r}$ :amplitude imbalance parameters

- $\theta_{t}, \theta_{r}$ :phase imbalance parameters

- $\xi_{t}^{I}(n), \xi_{r}^{I}(n)$ : I-branch LPF Impulse Response

- $\xi_{t}^{Q}(n), \xi_{r}^{Q}(n)$ : Q-branch LPF Impulse Response

The $\alpha$ 's and $\theta$ 's cause frequency-independent I/Q imbalance and $\xi$ 's result in frequency-dependent I/Q imbalance. Also, we denote the input signal as $\bar{s}(n)$, output signal as $\bar{z}(n)$, the additive white Gaussian noise (AWGN) as $\bar{w}(n)$ and the channel as $\bar{g}(n, l)$. The channel is double indexed to reflect its time-varying nature. The effect of the imbalances and channel could be summarized by Equation (1) where $N$ is the number of sub-carriers in the OFDM system, $\otimes$ represents the circular convolution operation and the $\lambda$ 's and $\phi$ 's are defined in the Table I. We use the overline notation to denote time-domain quantities. The $\bar{z}(n)$ 's could as well be described using the following matrix relation

$$
\begin{gathered}
\overline{\mathbf{z}}=\left(\overline{\boldsymbol{\Lambda}}_{r} \overline{\boldsymbol{\Lambda}}_{t} \overline{\mathbf{G}}+\overline{\boldsymbol{\Phi}}_{r} \overline{\boldsymbol{\Phi}}_{t}^{*} \overline{\mathbf{G}}^{*}\right) \overline{\mathbf{s}}+ \\
\left(\overline{\boldsymbol{\Lambda}}_{r} \overline{\boldsymbol{\Phi}}_{t} \overline{\mathbf{G}}+\overline{\mathbf{\Phi}}_{r} \overline{\boldsymbol{\Lambda}}_{t}^{*} \overline{\mathbf{G}}^{*}\right) \overline{\mathbf{s}}^{*}+\mathbf{v}
\end{gathered}
$$

where $\overline{\mathbf{v}}=\overline{\boldsymbol{\Lambda}}_{r} \overline{\mathbf{w}}+\overline{\mathbf{\Phi}}_{r} \overline{\mathbf{w}}^{*}$. The vectors $\overline{\mathbf{z}}, \overline{\mathbf{s}}, \overline{\mathbf{w}}$ are comprised of $\bar{z}(n), \bar{s}(n), \bar{w}(n)$, respectively, and $\overline{\boldsymbol{\Lambda}}_{t}, \overline{\boldsymbol{\Lambda}}_{r}, \overline{\mathbf{\Phi}}_{t}, \overline{\mathbf{\Phi}}_{r}, \overline{\mathbf{G}}$ are the matrices corresponding to $\bar{\lambda}_{t}(n), \bar{\lambda}_{r}(n), \bar{\phi}_{t}(n), \bar{\phi}_{r}(n), \bar{g}(n, l)$, respectively, of Equation (1). $\bar{\lambda}_{t}(n), \bar{\lambda}_{r}(n), \bar{\phi}_{t}(n), \bar{\phi}_{r}(n)$ do not vary with time and, hence, their corresponding matrices are circulant, whereas $\overline{\mathbf{G}}$ is not circulant since $\bar{g}(n, l)$ is time-varying. Applying DFT to (2) yields

$$
\begin{aligned}
& \mathbf{z}=\left(\boldsymbol{\Lambda}_{r} \boldsymbol{\Lambda}_{t} \mathbf{G}+\boldsymbol{\Phi}_{r} \boldsymbol{\Phi}_{t}^{\#} \mathbf{G}^{\#}\right) \mathbf{s}+ \\
& \left(\boldsymbol{\Lambda}_{r} \boldsymbol{\Phi}_{t} \mathbf{G}+\boldsymbol{\Phi}_{r} \boldsymbol{\Lambda}_{t}^{\#} \mathbf{G}^{\#}\right) \mathbf{s}^{\#}+\mathbf{v}
\end{aligned}
$$

where $\boldsymbol{\Lambda}_{t}=\mathbf{F} \overline{\boldsymbol{\Lambda}}_{t} \mathbf{F}^{H}, \boldsymbol{\Lambda}_{r}=\mathbf{F} \overline{\boldsymbol{\Lambda}}_{r} \mathbf{F}^{H}, \boldsymbol{\Phi}_{t}=\mathbf{F} \overline{\mathbf{\Phi}}_{t} \mathbf{F}^{H}$, $\boldsymbol{\Phi}_{r}=\mathbf{F} \overline{\boldsymbol{\Phi}}_{r} \mathbf{F}^{H}, \mathbf{G}=\mathbf{F} \overline{\mathbf{G}} \mathbf{F}^{H}, \mathbf{z}=\mathbf{F} \overline{\mathbf{z}}, \mathbf{s}=\mathbf{F} \overline{\mathbf{s}}$ and $\mathbf{v}=\mathbf{F} \overline{\mathbf{v}} . \mathbf{F}$ is the DFT matrix whose $(n, k)$-th element is given by $F_{n k}=\frac{1}{\sqrt{N}} e^{-j \frac{2 \pi k n}{N}} ; 0 \leq n, k \leq N-1$. The $k$-th element of a vector $\mathbf{b}^{\#}$ is related to the $k^{\prime}=(N-k)$ element of vector $\mathbf{b}$ by $b^{\#}(k)=b^{*}\left(k^{\prime}\right)$. Similarly, the $(n, k)$ element of a matrix $\mathbf{B}^{\#}$ is related to the $\left(n^{\prime}, k^{\prime}\right)=(N-n, N-k)$ element of matrix $\mathbf{B}$ by $b^{\#}(n, k)=b^{*}\left(n^{\prime}, k^{\prime}\right) . \boldsymbol{\Lambda}_{t}, \boldsymbol{\Lambda}_{r}, \boldsymbol{\Phi}_{t}, \boldsymbol{\Phi}_{r}$ are diagonal matrices since their corresponding time-domain matrices are circulant whereas $\mathbf{G}$ is not diagonal since $\overline{\mathbf{G}}$ is not circulant. 


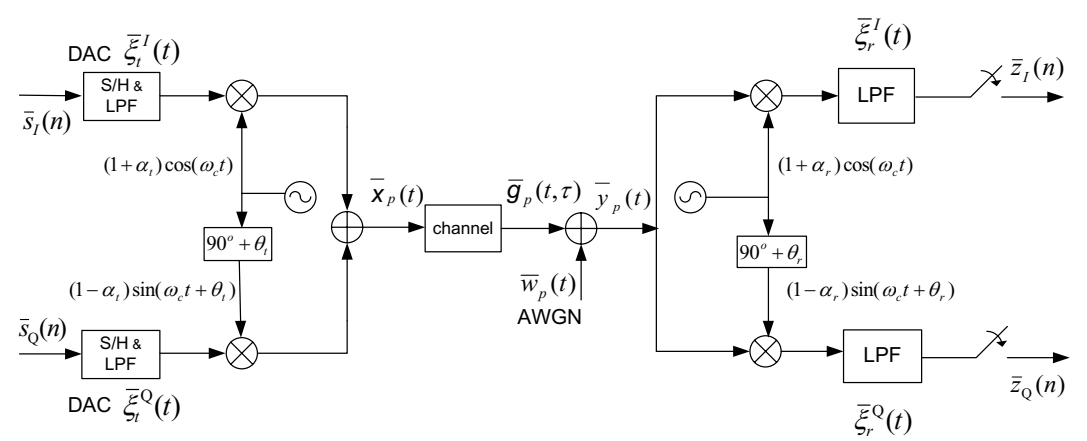

Fig. 1. OFDM System with I/Q imbalance

$$
\begin{aligned}
& \bar{z}(n)=\left[\bar{\lambda}_{r}(n) \otimes \bar{\lambda}_{t}(n) \otimes \bar{g}(n, l)+\bar{\phi}_{r}(n) \otimes \bar{\phi}_{t}^{*}(n) \otimes \bar{g}^{*}(n, l)\right] \otimes \bar{s}(n) \\
& +\left[\bar{\lambda}_{r}(n) \otimes \bar{\phi}_{t}(n) \otimes \bar{g}(n, l)+\bar{\phi}_{r}(n) \otimes \bar{\lambda}_{t}^{*}(n) \otimes \bar{g}^{*}(n, l)\right] \otimes \bar{s}^{*}(n) \\
& +\bar{\lambda}_{r}(n) \otimes \bar{w}(n)+\bar{\phi}_{r}(n) \otimes \bar{w}^{*}(n) ; 0 \leq n \leq N-1 .
\end{aligned}
$$

TABLE I

DEFINITION OF PARAMETERS IN EQUATION (1)

\begin{tabular}{|c|c|}
\hline Transmit-side & Receive-side \\
\hline $\bar{\lambda}_{t}(t)=\frac{1}{2}\left(\left(\mu_{t}+\nu_{t}\right) \bar{\xi}_{t}^{I}(t)+\left(\mu_{t}-\nu_{t}\right) \bar{\xi}_{t}^{Q}(t)\right)$ & $\bar{\lambda}_{r}(t)=\frac{1}{2}\left(\left(\mu_{r}+\nu_{r}^{*}\right) \bar{\xi}_{r}^{I}(t)+\left(\mu_{r}-\nu_{r}^{*}\right) \bar{\xi}_{r}^{Q}(t)\right)$ \\
\hline $\bar{\phi}_{t}(t)=\frac{1}{2}\left(\left(\mu_{t}+\nu_{t}\right) \bar{\xi}_{t}^{I}(t)-\left(\mu_{t}-\nu_{t}\right) \xi_{t}^{Q}(t)\right)$ & $\bar{\phi}_{r}(t)=\frac{1}{2}\left(\left(\nu_{r}+\mu_{r}^{*}\right) \bar{\xi}_{r}^{I}(t)+\left(\nu_{r}-\mu_{r}^{*}\right) \bar{\xi}_{r}^{Q}(t)\right)$ \\
\hline$\mu_{t}=\cos \left(\frac{\theta_{t}}{2}\right)-j \alpha_{t} \sin \left(\frac{\theta_{t}}{2}\right)$ & $\mu_{r}=\cos \left(\frac{\theta_{r}}{2}\right)+j \alpha_{r} \sin \left(\frac{\theta_{r}}{2}\right)$ \\
\hline$\nu_{t}=\alpha_{t} \cos \left(\frac{\theta_{t}}{2}\right)-j \sin \left(\frac{\theta_{t}}{2}\right)$ & $\nu_{r}=\alpha_{r} \cos \left(\frac{\theta_{r}}{2}\right)-j \sin \left(\frac{\theta_{r}}{2}\right)$ \\
\hline
\end{tabular}

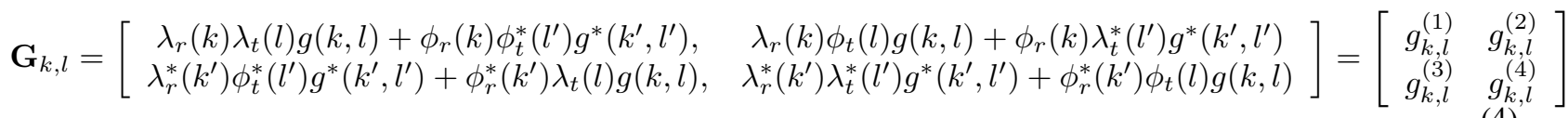

$$
\begin{aligned}
& \mathbf{G}_{k, l}^{(I m)}=\left[\begin{array}{cc}
\lambda_{r}(k) \phi_{t}\left(l^{\prime}\right) g\left(k, l^{\prime}\right)+\phi_{r}(k) \lambda_{t}^{*}(l) g^{*}\left(k^{\prime}, l\right), & \lambda_{r}(k) \lambda_{t}\left(l^{\prime}\right) g\left(k, l^{\prime}\right)+\phi_{r}(k) \phi_{t}^{*}(l) g^{*}\left(k^{\prime}, l\right) \\
\lambda_{r}^{*}(k) \lambda_{t}^{*}(l) g^{*}\left(k^{\prime}, l\right)+\phi_{r}^{*}\left(k^{\prime}\right) \phi_{t}\left(l^{\prime}\right) g\left(k, l^{\prime}\right), & \lambda_{r}^{*}\left(k^{\prime}\right) \phi_{t}^{*}(l) g^{*}\left(k^{\prime}, l\right)+\phi_{r}^{*}\left(k^{\prime}\right) \lambda_{t}\left(l^{\prime}\right) g\left(k, l^{\prime}\right)
\end{array}\right] \\
& \tilde{\mathbf{G}}(k)=\left[\begin{array}{ccccccc}
\mathbf{G}_{k-D, k-2 D} & \cdots & \mathbf{G}_{k-D, k-D} & \ldots & \mathbf{G}_{k-D, k} & & \\
& \mathbf{G}_{k, k-D} & \cdots & \mathbf{G}_{k, k} & \cdots & \mathbf{G}_{k, k+D} & \\
& & \mathbf{G}_{k+D, k} & \cdots & \mathbf{G}_{k+D, k+D} & \cdots & \mathbf{G}_{k+D, k+2 D}
\end{array}\right]
\end{aligned}
$$

Equalization of $\mathbf{z}$ involves the inversion of a matrix of size $N \times N$ which is computationally complex for large $N$. Therefore, we derive the following approximate model. Focussing on the $k$ and $k^{\prime}=N-k$ (a sub-carrier and its image) elements of $\mathbf{z}$ in Equation (3), we can write

$$
\begin{aligned}
\mathbf{z}_{k} & =\mathbf{G}_{k, k} \mathbf{s}_{k}+\sum_{l=0, l \neq k}^{\frac{N}{2}-1} \mathbf{G}_{k, l} \mathbf{s}_{l}+\sum_{l=0}^{\frac{N}{2}-1} \mathbf{G}_{k, l}^{(I m)} \mathbf{s}_{l}^{*} \\
& +\mathbf{v}_{k} ; 0 \leq k \leq \frac{N}{2}-1
\end{aligned}
$$

where $\mathbf{z}_{k}=\left[z_{k} ; z_{k^{\prime}}^{*}\right]^{T}, \mathbf{s}_{k}=\left[s_{k} ; s_{k^{\prime}}^{*}\right]^{T}, \mathbf{v}_{k}=\left[v_{k} ; v_{k^{\prime}}^{*}\right]^{T}$, $\mathbf{G}_{k, l}$ is defined in (4) and $\mathbf{G}_{k, l}^{(I m)}$ defined in (5). The term $\mathbf{G}_{k, l}^{(I m)}$ represents self-interference occurring due to the combined effects of mobility and I/Q imbalance. For a symbol $s(k)$ at sub-carrier $k$, I/Q imbalance results in an image term at sub-carrier $k^{\prime}$ and due to mobility, a component of $s(k)$ again interferes with itself at sub-carrier $k$ in conjugate form. But it should be noted that even for very high Doppler spreads, a sub-carrier interferes only with some of its adjacent sub-carriers. Let's say it affects only $D$ sub-carriers on either side, then only those $g(k, l)$ for which $|k-l|<D$ need to be considered and the rest can be set to zero. This implies that $\mathbf{G}_{k, l}=\mathbf{0}_{2 \times 2}$ for $|k-l|>D$ and $\mathbf{G}_{k, l}^{(I m)}=\mathbf{0}_{2 \times 2} \forall k, l$. Hence we can write 
the simplified approximate model

$\mathbf{z}_{k}=\mathbf{G}_{k, k} \mathbf{s}_{k}+\sum_{l=k-D, l \neq k}^{k+D} \mathbf{G}_{k, l} \mathbf{s}_{l}+\mathbf{v}_{k} ; 0 \leq k \leq \frac{N}{2}-1$.

\section{Compensation Schemes}

We exploit the banded structure of $\mathbf{G}$ and arrive at a low-complexity equalizer described as follows. We develop the equalizer for the case of $Q=3$ and extension to larger values of $Q$ is straight-forward. First, we define the following quantities based on (8)

$\tilde{\mathbf{z}}_{k}=\left[\begin{array}{c}\mathbf{z}_{k-D} \\ \vdots \\ \mathbf{z}_{k} \\ \vdots \\ \mathbf{z}_{k+D}\end{array}\right], \tilde{\mathbf{s}}_{k}=\left[\begin{array}{c}\mathbf{s}_{k-2 D} \\ \vdots \\ \mathbf{s}_{k} \\ \vdots \\ \mathbf{s}_{k+2 D}\end{array}\right], \tilde{\mathbf{v}}_{k}=\left[\begin{array}{c}\mathbf{v}_{k-D} \\ \vdots \\ \mathbf{v}_{k} \\ \vdots \\ \mathbf{v}_{k+D}\end{array}\right]$,

and $\tilde{\mathbf{G}}(k)$ as in (6). Then, we have

$$
\tilde{\mathbf{z}}_{k}=\tilde{\mathbf{G}}_{k} \tilde{\mathbf{s}}_{k}+\tilde{\mathbf{v}}_{k} .
$$

The MMSE estimate for $\mathbf{s}_{k}$ is given by

$$
\hat{\mathbf{s}}_{k}=\mathbf{w}_{k}^{H} \tilde{\mathbf{z}}_{k}
$$

where $\mathbf{w}_{k}^{H}=\overline{\tilde{\mathcal{G}}}_{k}^{H}\left(\tilde{\mathbf{G}}_{k} \tilde{\mathbf{G}}_{k}^{H}+\sigma_{v}^{2} \mathbf{I}_{6}\right)^{-1}$ with $\tilde{\mathcal{G}}_{k}=$ $\left[\mathbf{G}_{k-D, k}^{T}, \cdots, \mathbf{G}_{k+D, k}^{T^{k}}\right]^{T}$. Note that using (10), we detect the $k$-th sub-carrier symbol $s_{k}$ and its image $s_{k^{\prime}}^{*}$ jointly.

\section{Channel Estimation Scheme}

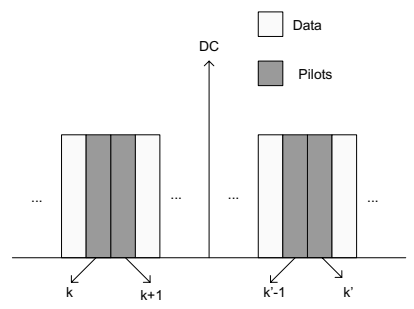

Fig. 2. Pilot Pattern

In order to perform the equalization step in (10), we need to estimate the $\mathbf{G}_{k, l}$ 's in (6). To do this, we first ignore the ICI due to mobility and estimate only the $\mathbf{G}_{k, k}$ 's. This does not affect the results significantly if pilots are boosted in power relative to the data subcarriers. Ignoring the terms in the summation, we note that equation (8) can be rewritten as

$$
\mathbf{z}_{k}=\left[\begin{array}{cccc}
s_{k} & 0 & s_{k^{\prime}}^{*} & 0 \\
0 & s_{k} & 0 & s_{k^{\prime}}^{*}
\end{array}\right] \underbrace{\left[\begin{array}{c}
g_{k, l}^{(1)} \\
g_{k, l}^{(2)} \\
g_{k, l}^{(3)} \\
g_{k, l}^{(4)}
\end{array}\right]}_{\mathbf{h}_{k}}+\mathbf{v}_{k}
$$

We do not have to solve for each term in $\mathbf{h}_{k}$, but consider each row as one unknown and solve for it. This is a system with two equations and four unknowns. To obtain two more equations, we use a pilot scheme as shown in Fig. 2 wherein we have pairs of pilots on adjacent sub-carriers and their images. The channel frequency response and the frequency-selective I/Q imbalance parameters can be considered equal over two adjacent subcarriers. Under this assumption, we can write

$$
\mathbf{z}_{k}=\underbrace{\left[\begin{array}{cccc}
s_{k} & 0 & s_{k^{\prime}}^{*} & 0 \\
0 & s_{k} & 0 & s_{k^{\prime}}^{*} \\
s_{k+1} & 0 & s_{k^{\prime}-1}^{*} & 0 \\
0 & s_{k+1} & 0 & s_{k^{\prime}-1}^{*}
\end{array}\right]}_{\mathbf{S}_{k}}\left[\begin{array}{c}
g_{k, l}^{(1)} \\
g_{k, l}^{(2)} \\
g_{k, l}^{(3)} \\
g_{k, l}^{(4)}
\end{array}\right]+\mathbf{v}_{k}
$$

Now, $\mathbf{h}_{k}$ can be computed using from (12) using Least Squares estimation. Once the $\mathbf{G}_{k, k}$ 's have been obtained, the off-diagonal elements $\left(\mathbf{G}_{k, l}\right.$ 's with $\left.k \neq l\right)$ can be obtained using the method described in [10]. Basically, this involves going to the time-domain and exploiting the correlations of channel taps across time using linear interpolation.

\section{EXTENSION TO MIMO SM-OFDM SYSTEMS}

In this section, we study the effects of I/Q imbalance and mobility on MIMO-OFDM, in particular, SMOFDM systems. We consider an SM-OFDM system with $N_{Q}$ transmit and $N_{P}$ receive antennas with $N_{P} \geq N_{Q}$ to enable low-complexity linear MMSE decoding. To derive the model for an SM-OFDM system with impairments, we apply the same signal-flow model in Fig. 1 , but that one such chain need to be assumed between each transmit-receive antenna pair with a different set of parameters (in general). Within each sub-carrier, a different symbol is transmitted over each transmit antenna. We use the notation $s_{q}(k)$ to denote the symbol transmitted from antenna- $q$ of sub-carrier $k$. Then, using (8), we can write for the symbols received at antenna $p$ of sub-carriers $k$ and $k^{\prime}$,

$$
\mathbf{z}_{k}(p)=\sum_{q=0}^{N_{Q}-1} \sum_{l=k-D}^{k+D} \mathbf{G}_{k, l}(p, q) \mathbf{s}_{l}(q)+\mathbf{w}_{k}(p)
$$


where $\mathbf{z}_{k}(p)=\left[z_{k}(p) z_{k^{\prime}}^{*}(p)\right]^{T}, \mathbf{s}_{l}(q)=\left[s_{l}(q) s_{l^{\prime}}^{*}(q)\right]^{T}$, $\mathbf{w}_{k}(p)=\left[w_{k}(p) w_{k^{\prime}}^{*}(p)\right]^{T}$,

$$
\mathbf{G}_{k, l}(p, q)=\left[\begin{array}{cc}
g_{k, l}^{(1)}(p, q) & g_{k, l}^{(2)}(p, q) \\
g_{k, l}^{(3)}(p, q) & g_{k, l}^{(4)}(p, q)
\end{array}\right] .
$$

Index $p$ runs from 0 to $N_{P}-1$, and index $k$ runs from 0 to $\frac{N}{2}-1$. The quantities $g_{k, l}^{(i)}(p, q) ; i=1,2,3,4$ are similar to the ones in (4) except for the antenna indices. In an SM-OFDM system, the symbols for the set of all receive antennas are grouped together for detection. For this purpose, we define $\mathbf{z}_{k}=\left[\mathbf{z}_{k}^{T}(0), \cdots, \mathbf{z}_{k}^{T}\left(N_{P}-\right.\right.$ $1)]^{T}, \mathbf{s}_{k}=\left[\mathbf{s}_{k}^{T}(0), \cdots, \mathbf{s}_{k}^{T}\left(N_{Q}-1\right)\right]^{T}$ and $\mathbf{w}_{k}=$ $\left[\mathbf{w}_{k}^{T}(0), \cdots, \mathbf{w}_{k}^{T}\left(N_{P}-1\right)\right]^{T}$. Then, we can write

$$
\mathbf{z}_{k}=\sum_{l=k-D}^{k+D} \mathbf{G}_{k, l} \mathbf{s}_{l}+\mathbf{w}_{k}
$$

where

$$
\mathbf{G}_{k, l}=\left[\begin{array}{ccc}
\mathbf{G}_{k, l}(0,0) & \cdots & \mathbf{G}_{k, l}\left(0, N_{Q}-1\right) \\
\vdots & \ddots & \vdots \\
\mathbf{G}_{k, l}\left(N_{P}-1,0\right) & \cdots & \mathbf{G}_{k, l}\left(N_{P}-1, N_{Q}-1\right)
\end{array}\right]
$$

From (14), it can be seen that to detect $\mathbf{s}_{k}$, the sufficient statistic is provided by $\tilde{\mathbf{z}}_{k}=\left[\mathbf{z}_{k-D}^{T}, \cdots, \mathbf{z}_{k+D}^{T}\right]^{T}$. Morevoer, if we define $\tilde{\mathcal{G}}_{k}=\left[\mathbf{G}_{k-D, k}^{T}, \cdots, \mathbf{G}_{k+D, k}^{T}\right]^{T}$ and $\tilde{\mathbf{G}}_{k}$ as in (6), the linear MMSE estimate of $\mathbf{s}_{k}$ is given by

$$
\hat{\mathbf{s}}_{k}=\tilde{\mathcal{G}}_{k}^{H}\left(\tilde{\mathbf{G}}_{k} \tilde{\mathbf{G}}_{k}^{H}+\frac{1}{S N R} \mathbf{I}_{2(2 D+1) N_{P}}\right)^{-1} \tilde{\mathbf{z}}_{k}
$$

The channel estimation procedure for the MIMO case is similar to that of the SISO case, but here four different parameters need to be estimated for each transmitreceive antenna pair. Consider the case where we have an equal number of transmit and receive antennas, say $N_{P}$. Since there are $N_{P}^{2}$ antenna pars, we will have $4 N_{P}^{2}$ unknowns in all to be estimated. Therefore, we need as many equations and, hence, as many pilots. If the $\mathrm{I} / \mathrm{Q}$ imbalance is frequency independent and if the the I/Q imbalance parameters ( $\mu$ 's and $\nu$ 's) for all the antennas on either the transmitter or receiver side can be considered equal, then the number of unknowns is reduced and hence we can also reduce the number of pilots required. To get the off-diagaonal elements $\left(\mathbf{G}_{k, l}(p, q)\right.$ 's with $\left.k \neq l\right)$, the procedure used in the SISO case is performed for every trasmit-receive antenna pair.

\section{Simulation Results}

Simulation results for SISO and MIMO OFDM systems are provided in this section. A 512-subcarrier OFDM system with a $5 \mathrm{MHz}$ bandwidth is used over a fading channel based on SUI-3 model with a delay spread of $10 \mu \mathrm{s}$. A rate- $\frac{1}{2}$ convolutional code (gener- ator polynomial $(171,133))$ with random interleaving is also used. The I/Q imbalance parameters used are $\alpha_{r}=\alpha_{t}=0.5 \mathrm{~dB}, \theta_{r}=\theta_{t}=5^{\circ}$ and the Doppler spread is $5 \%$ of sub-carrier spacing corresponding to an user velocity of $235 \mathrm{kmph}$. For frequency-depedent I/Q imbalance, we use the filter parameters $\bar{\xi}_{I}^{t}(t)=$ $\bar{\xi}_{I}^{r}(n)=0.01 \delta(n)+\delta(n-1)+0.01 \delta(n-2)$ and $\bar{\xi}_{Q}^{t}(t)=\bar{\xi}_{Q}(n)=0.01 \delta(n)+\delta(n-1)+0.2 \delta(n-2)$. Fig. 3 shows the BER comparisons for the SISO case employing 16-QAM for both frequency-dependent and frequency-independent I/Q imbalance cases with mobility. Clearly, there is an error floor and the proposed equalization scheme significantly improves performance. The BER performance curve for the estimated channel case is only slightly inferior to the perfect CSI case. Fig. 4 shows the results for the 64-QAM constellation, where the performance varies significantly with as we go from $Q=3$ to $Q=5$.

Shown in Fig. 5 is a similar set of BER curves for SM-OFDM case with 2-transmit and 2-receive antennas, but the I/Q imbalance is assumed to be freuqencyindependent. Again, it is evident from the simulations that our proposed digital baseband compensation scheme results in substantial performance improvements. We conclude by mentioning that we also developed estimation and compensation schemes for MIMOOFDM transceivers that use space-frequency block coding (SFBC) to achieve diversity gains (see [11] for more details).

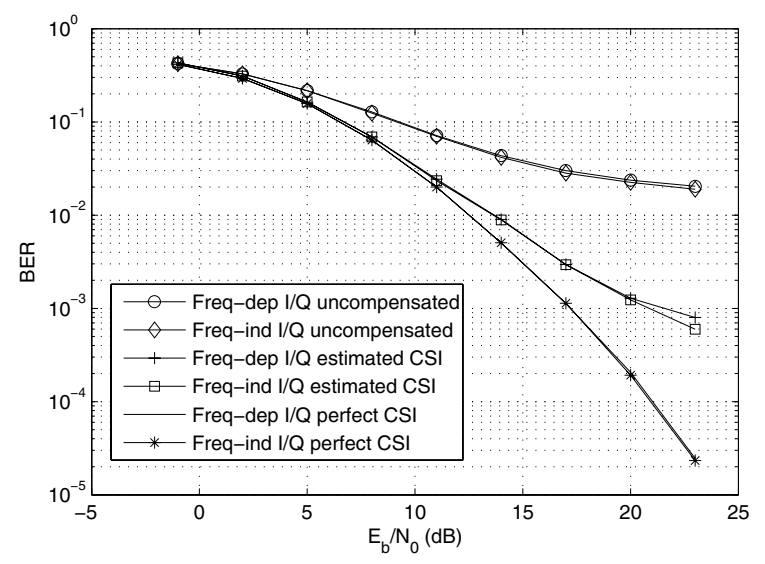

Fig. 3. Results for 16-QAM with frequency dependent $\mathrm{I} / \mathrm{Q}$ imbalance. $\bar{\xi}_{I}^{t}(t)=\bar{\xi}_{I}^{r}(n)=0.01 \delta(n)+\delta(n-1)+0.01 \delta(n-2), \bar{\xi}_{Q}^{t}(t)=$ $\bar{\xi}_{Q}(n)=0.01 \delta(n)+\delta(n-1)+0.2 \delta(n-2)$

\section{CONCLUSIONS}

In this work, we developed generalized baseband models for both frequency-dependent and frequencyindependent joint transmit-receive I/Q imbalance for mobile SISO and MIMO OFDM transceivers. Based on 


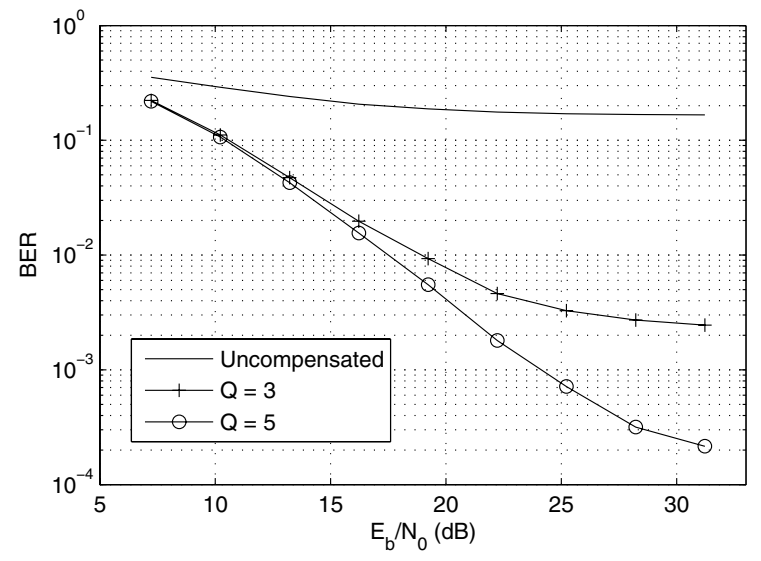

Fig. 4. Results for 64-QAM with frequency-independent I/Q imbalance assuming perfect CSI

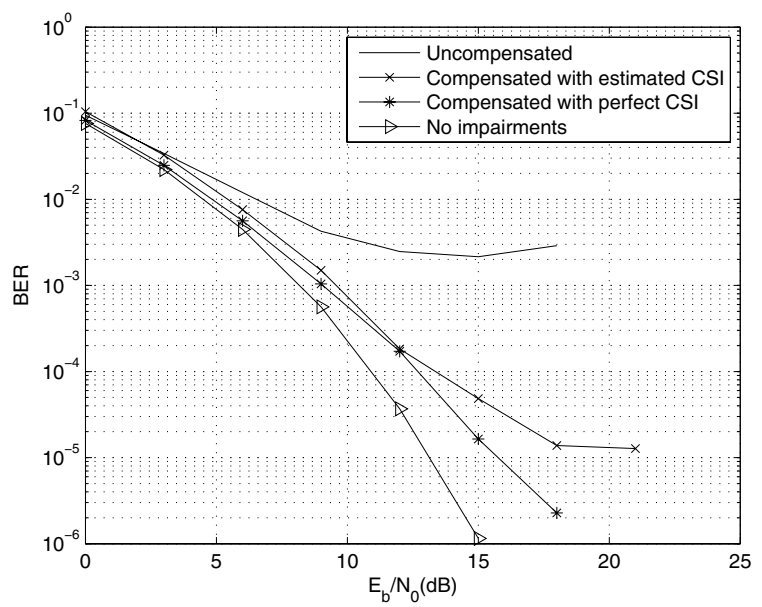

Fig. 5. Results for 4-QAM SM-OFDM and frequency independent I/Q imbalance

these models, we proposed low-complexity digital baseband compensation schemes to mitigate the ICI induced by mobility and I/Q imbalance. We also addressed the issue of channel and I/Q imbalance parameter estimation by proposing a novel pilot scheme. Our simulation results showed that our proposed digital baseband compensation schemes achieve significant performance improvements compared to the uncompensated system.

\section{ACKNOWLEDGMENT}

The authors would like to thank Dandan Wang for her valuable comments and suggestions.

\section{REFERENCES}

[1] C. L. Liu, "Impacts of I/Q imbalance on QPSK-OFDM-QAM detection," IEEE Trans. Consum. Electron., vol. 44, no. 3, pp. 984-989, Aug. 1998.
[2] A. Schuchert, R. Hasholzne, and P. Anotine, "A novel I/Q imbalance compensation scheme for the reception of OFDM signals," IEEE Trans. Consum. Electron., vol. 47, no. 3, pp. 313$318,2001$.

[3] A. Tarighat, R. Bagheri, and A. H. Sayeed, "Compensation schemes and peformance analysis of IQ imbalances in OFDM receivers," IEEE Trans. Signal Process., vol. 53, no. 8, pp. 32573268, Aug. 2005.

[4] A. Tarighat and A. H. Sayed, "Joint compensation of transmitter and receiver impairments in OFDM systems," IEEE Transactions on Wireless Communications, vol. 6, no. 1, pp. 240-247, Jan. 2007.

[5] M. Valkama, M. Renfors, and V. Koivunen, "Compensation of frequency-selective I/Q imbalances in wideband receivers: models and algorithms," in IEEE Third Workshop on Signal Processing Advances in Wireless Communications (SPAWC '01), Mar. 2001, pp. 42-45.

[6] J. Tubbax, B. Come, L. V. der Perre, L. Deneire, S. Donnay, and M. Engels, "Compensation of IQ imbalance in OFDM systems," in IEEE ICC, vol. 5, May 2003, pp. 3403 - 3407.

[7] P. Rykaczewski, J. Brakensiek, and F. Jondral, "Decision directed methods of I/Q imbalance compensation in OFDM systems," in IEEE VTC, vol. 1, Sept. 2004, pp. $484-487$.

[8] D. Tandur and M. Moonen, "Joint adaptive compensation of transmitter and receiver IQ imbalance under carrier frequency offset in OFDM-based systems," IEEE Trans. Signal Process., vol. 55, no. 11, pp. 5246 - 5252, Nov. 2007.

[9] B. Narasimhan, S. Narayanan, N. Al-Dhahir, and H. Minn, "Digital baseband compensation of I/Q imbalance in mobile OFDM," IEEE WCNC, pp. 1183-1187, Mar. 2008.

[10] Y. Mostofi and D. C. Cox, "ICI mitigation for pilot-aided OFDM mobile systems," IEEE Trans. Wireless Commun., vol. 4, pp. 765774, Mar. 2005.

[11] B. Narasimhan, D. Wang, S. Narayanan, N. Al-Dhahir, and H. Minn, "Digital baseband compensation for mobile SFBCOFDM systems with receiver I/Q imbalance," IEEE Globecom, Dec. 2008. 